\title{
CARACTERÍSTICAS E ALTERAÇÕES NEUROPSICOLÓGICAS NO TRANSTORNO DE PERSONALIDADE BORDERLINE: UMA REVISÃO DA LITERATURA
}

\author{
NEUROPSYCHOLOGICAL CHARACTERISTICS AND CHANGES IN BORDERLINE \\ PERSONALITY DISORDER: A LITERATURE REVIEW
}

\author{
Rodrigo Barbosa Nascimento \\ Gabriela de Lima Cerqueira ${ }^{2}$ \\ Emanuel Santos de Araujo Filho ${ }^{3}$
}

\begin{abstract}
RESUMO: A neuropsicologia é uma área de estudo que engloba os aspectos físicos e cognitivos; portanto, é possível investigar as diversas nuances que envolvem os transtornos psiquiátricos, sobretudo o Transtorno de Personalidade Borderline (TPB). OBJETIVO: Este estudo objetivou apresentar evidências científicas e sistematizar as principais compreensões da literatura científica publicadas entre 2010 e 2021 acerca das características e alterações neuropsicológicas presentes no TPB. MÉTODO: Durante os meses de fevereiro e março de 202I, realizou-se uma revisão integrativa da literatura científica acerca da temática em questão, sendo utilizados os descritores indexados no DeCS: Transtorno da Personalidade Borderline; Transtorno da Personalidade Limítrofe; Neuropsicologia; Neurobiologia. Para realizar a pesquisa, os descritores foram combinados nas bases de dados SciELO, PepsiC, pubMED/Medline e BVS/Lilacs. RESULTADOS: Associadas ao TPB, foram identificadas alterações a nível cognitivo, assim como alterações em estrutura e função nas regiões (amígdala, hipocampo e hipotálamo) que comportam o sistema límbico do cérebro à outras alterações externas a esse sistema. A nível cognitivo destacam-se: menor funcionamento adaptativo, alterações na memória, atenção e no processamento emocional. $\mathrm{Na}$ amígdala evidencia-se: o aumento das ativações em contraste com a redução e a diminuição do volume. Já nos outros dois, redução do volume do primeiro em contraste com o aumento do segundo. Externa à região límbica ressaltam-se alterações nas regiões de controle pré-frontal e a maior concentração de $\mathrm{N}$-acetilasparto e glutamato. CONCLUSÃO: O TPB apresenta alterações a nível cognitivo e a nível biológico; no entanto, tornam-se necessárias maiores investigações acerca da inter-relação entre essas alterações.
\end{abstract}

Palavras-chave: Transtorno da Personalidade Borderline. Transtorno da Personalidade Limítrofe. Neuropsicologia. Neurobiologia.

\footnotetext{
I Acadêmico do curso de Psicologia da Escola de Ciências da Saúde e Bem-estar da Universidade Salvador Laureate International Universities (UNIFACS), Feira de Santana - BA. Membro do Núcleo de Estudos em Comportamento, Filosofia e Psicanálise (NECOFIP). E-mail: nascimentolag@hotmail.com.

${ }^{2}$ Acadêmica do curso de Psicologia da Escola de Ciências da Saúde e Bem-estar da Universidade Salvador Laureate International Universities (UNIFACS), Feira de Santana - BA. Membro do Núcleo de Estudos em Comportamento, Filosofia e Psicanálise (NECOFIP).E-mail: gabipopyrors@gmail.com.

3 Acadêmico do curso de Psicologia da Escola de Ciências da Saúde e Bem-estar da Universidade Salvador Laureate International Universities (UNIFACS), Feira de Santana - BA. Membro do Núcleo de Estudos em Comportamento, Filosofia e Psicanálise (NECOFIP). E-mail: emanuelfilho6ı6@gmail.com.
} 
ABSTRACT: Neuropsychology is an area of study that encompasses the physical and cognitive aspects; therefore, it is possible to investigate the various nuances that involve psychiatric disorders, especially Borderline Personality Disorder (TPB). OBJECTIVE: This study aimed to present scientific evidence and systematize the main understandings of the scientific literature published between 2010 and 2021 about the neuropsychological characteristics and changes present in the TPB. METHOD: During the months of February and March 2020, an integrative review of the scientific literature was carried out on the subject in question, using the descriptors indexed in the DeCS: Borderline Personality Disorder; Borderline Personality Disorder; Neuropsychology; Neurobiology. To carry out the research, the descriptors were combined in the SciELO, PepsiC, pubMED / Medline and BVS / Lilacs databases. RESULTS: Associated with TPB, changes were identified at the cognitive level, as well as changes in structure and function in the regions (amygdala, hippocampus and hypothalamus) that involve the limbic system of the brain to other changes external to that system. At the cognitive level, the following stand out: less adaptive functioning, changes in memory, attention and emotional processing. In the amygdala it is evident: the increase in activations in contrast to the reduction and decrease in volume. In the other two, there was a reduction in the volume of the first, in contrast to the increase in the second. External to the limbic region, changes in the prefrontal control regions and the higher concentration of $\mathrm{N}$-acetylsparto and glutamate are highlighted. CONCLUSION: TPB presents alterations at a cognitive and biological level; however, further investigations about the interrelationship between these changes are necessary.

Keywords: Borderline Personality Disorder. Neuropsychology. Neurobiology.

\section{INTRODUÇÃO}

A Neuropsicologia é uma disciplina de interseção baseada nas contribuições da neurologia, psicologia cognitiva e da psiquiatria (Stirling \& Elliot, 2008). Segundo Luria (198I), ela emergiu como um instrumento e saber eficaz para a compreensão e revisão dos conceitos substanciais sobre a estrutura interna de processos psicológicos e como fator essencial para a criação de uma teoria de base cerebral da atividade mental humana.

De acordo com Dalgalarrondo (2019), a neuropsicologia é uma área que estuda as funções neurobiológicas e os impactos relacionados às alterações de nível estrutural e funcional em correlação com os aspectos cognitivos. Em outras palavras, é um ramo da ciência que objetiva a investigação do papel dos sistemas cerebrais individuais em formas complexas da atividade mental (Luria, 198I, p. 4), que, por sua vez, dentre seus focos de investigação aparecem as alterações presentes nos demais transtornos mentais, isto é, as características e alterações neuropsicológicas que constituem um determinado transtorno.

Conforme o Manual Diagnóstico e Estatístico dos Transtornos Mentais - DSM 5, um transtorno é o conjunto de sinais e sintomas que promovem perturbações cognitivas, emocionais ou comportamentais que ocasionam sofrimento clinicamente significativo, em que seu fator etiológico é eminentemente multifatorial (APA, 2014, p. 20). Posto isso, um único fator não é suficiente para determinar a produção de um transtorno, mas pode ser o 
seu desencadeante quando o sujeito apresenta condições pré-existentes (psíquicas, genéticas, neurobiológicas, ambientais e sociais).

O transtorno de personalidade é um padrão contínuo de experiências e comportamentos internos que difere muito das expectativas culturais e pessoais, em especial por sua característica difusa e rígida (APA, 2014, p. 645). Os transtornos de personalidade, de maneira geral, são considerados bastante frequentes pela epidemiologia psiquiátrica e, dentre eles, destaca-se o transtorno de personalidade borderline (Chapman, Jamil \& Fleisher, 2021).

O transtorno de personalidade borderline ou transtorno de personalidade limítrofe (TPB) é caracterizado por uma dificuldade acentuada em estabelecer e manter relações interpessoais paralelo a comportamentos impulsivos que normalmente geram sofrimento ao próprio sujeito e aos seus companheiros (APA, 2014, p. 663). As principais características desse transtorno destacam a mudança de humor exacerbada, instabilidade em relações afetivas, sentimentos de abandono e vazio.

Assim, uma vez que o TPB está imerso na lógica multifatorial dos transtornos, emerge a necessidade de se falar e compreender acerca das alterações cognitivas, assim como alterações a nível neurobiológico, tanto em função quanto em estrutura. Sendo assim, devido a uma notável escassez de produções brasileiras relacionadas ao transtorno de personalidade borderline e suas alterações do ponto de vista neuropsicológico/neurobiológico, esse artigo se configura como de extrema importância para o desenvolvimento desse conhecimento na literatura brasileira, haja vista que integra os achados mais recentes sobre a temática em questão, sobretudo oriundos de outras línguas.

Desta forma, a partir de uma revisão integrativa, esse artigo objetivou sistematizar as principais compreensões da literatura científica publicadas entre 2010 e 2021 acerca das características e alterações neuropsicológicas presentes no transtorno de personalidade borderline, no período de fevereiro e março de 2021.

\section{Método}

A partir da construção de uma revisão integrativa, esse estudo se propôs a demonstrar sistematicamente evidências científicas acerca das principais características e alterações neuropsicológicas que estão sendo apresentadas e debatidas no que tange ao Transtorno de Personalidade Borderline (TPB). 
Esse tipo de revisão foi devidamente escolhido por seu caráter metodológico eminentemente mais definido e, sobretudo, ampliado em pesquisas de literatura, dado que abre a possibilidade para a inserção de artigos observacionais e experimentais, assim como estudos de revisão (Souza, Silva \& Carvalho, 20ıo).

O primeiro passo para o desenvolvimento da presente revisão partiu da construção da pergunta norteadora: "Quais as características e alterações neuropsicológicas e neurobiológicas podem ser encontradas no Transtorno de Personalidade Borderline?”. Em seguida, foi feito um levantamento no DeCS (Descritores em Ciências da Saúde), utilizando os descritores: Transtorno da Personalidade Borderline; Transtorno da Personalidade Limítrofe; Neuropsicologia; Neurobiologia; e seus correspondentes na língua inglesa. Para a pesquisa, realizaram-se os seguintes cruzamentos: "Transtorno da Personalidade Borderline" or "Transtorno da Personalidade Limítrofe" and "Neuropsicologia" or "Neurobiologia". O levantamento da amostra para a realização desse estudo foi realizado nas bases de dados SciELO, PepsiC, pubMED/Medline e BVS/Lilacs, no período de fevereiro e março de 202I.

A eleição dos estudos deu-se por meio dos seguintes critérios de inclusão: Artigos originais; Artigos de revisão; Ensaios clínicos que apresentam acerca das características neurobiológicas/neuropsicológicas do transtorno borderline, sem restrição quanto a língua. Além disso, foram incluídos apenas artigos publicados entre o período de 2010 e 202I.

Como critérios de exclusão optou-se por excluir: Ensaios teóricos; relatos de experiência; livros; monografias; teses; dissertações; resenhas de livros; tradução de artigos; artigos de intervenções neuropsicológicas ou programa de tratamento; bem como artigos sem clareza metodológica, ou seja, artigos que não apresentavam objetivo ou conclusão de maneira clara ou condizente com a proposta do estudo em questão.

No processo de busca (identificação), primeiramente foram achados 8I artigos no total, dos quais 5 foram eliminados por repetição no início da triagem. Após isso, prosseguindo com a triagem, 56 estudos foram excluídos após a leitura dos títulos e resumo, sendo descartados aqueles que não apresentavam as características e/ou alterações neuropsicológicas/neurobiológicas presentes no TPB $(n=27)$ ou apresentavam alterações e características neuropsicológicas/neurobiológicas de outros transtornos, assim como aqueles que demonstravam alterações neuropsicológicas/neurobiológicas do TPB no 
processo de alguma intervenção terapêutica $(n=15)$. Seguidamente, foram considerados elegíveis 20 artigos restantes.

Por fim, os 20 estudos foram lidos integralmente, sendo excluído o após análise da qualidade metodológica dos estudos, isto é, os 20 artigos restantes apresentavam de maneira clara as características e alterações neuropsicológicas e neurobiológicas do TPB, assim como indicação do objetivo e consideração final que corroboravam com o intuito desse estudo. Todo esse processo está exemplificado na Figura I.

Figura 1 - Fluxograma de triagem e estratégia de busca dos artigos

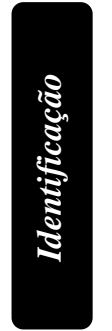

Estudos identificados através de pesquisas nas bases de dados

SciELO: 1; BVS/Lilacs: 19; PepsiC: 0; PubMED/Medline: 61

$$
\mathrm{N}=81
$$

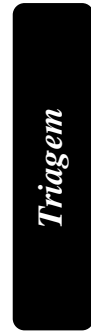

Estudos consideráveis elegíveis

$\mathrm{N}=20$

Estudos incluídos nesta revisão
Estudos adicionais identificados através de outras fontes

$$
\mathrm{N}=0
$$

56 estudos excluídos após a leitura dos títulos e resumos

Não apresentavam as características e/ou alterações neuropsicológicas/neurobiológicas presentes no TPB $(n=29)$

Apresentavam alterações e características neuropsicológicas/neurobiológicas de outro transtorno $(\mathrm{n}=11)$

Demonstravam alterações neuropsicológicas /neurobiológicas do TPB no processo de alguma intervenção terapêutica $(n=16)$.

\section{0 estudos excluídos após a leitura do texto na} íntegra

$$
\mathrm{N}=20
$$




\section{Resultados}

A revisão se constituiu através da coleta de 20 publicações (Quadro I) acerca das características e alterações neuropsicológicas/neurobiológicas presentes no Transtorno de personalidade Borderline.

No Quadro I, é possível identificar quais tipos de estudos foram mais encontrados para o desenvolvimento do presente artigo, destes $45 \%$ são artigos de revisão e $55 \%$ são ensaios clínicos.

Ainda no Quadro I, torna-se evidente um quantificado maior de artigos indexados no pubMED/Medline (90\%), prosseguidos pela BVS/Lilacs (5\%) e SciELO (5\%). Por motivos de transparência, é valido de menção que, embora o BVS/Lilacs apresente apenas $5 \%$ do total de artigos, os artigos dessa base de dados se encontravam em repetição com aqueles achados no pubMED/Medline.

Quadro I. Categorização dos artigos que apresentam características e/ou alterações neuropsicológicas/neurobiológicas presente no TPB.

\begin{tabular}{|c|c|c|c|c|c|c|}
\hline № & $\begin{array}{c}\text { AUTOR } \\
\text { (ES) }\end{array}$ & TÍTULO & INDEXADOR & $\begin{array}{c}\text { TIPO } \\
\text { DO } \\
\text { ESTUDO }\end{array}$ & PERIODICO & ANO \\
\hline I & $\begin{array}{c}\text { Bertsch K; } \\
\text { Hillmann } \\
\text { K; Herpertz } \\
\text { SC. }\end{array}$ & $\begin{array}{c}\text { Behavioral and } \\
\text { Neurobiological } \\
\text { Correlates of } \\
\text { Disturbed Emotion } \\
\text { Processing in } \\
\text { Borderline } \\
\text { Personality } \\
\text { Disorder. }\end{array}$ & pubMED/Medline & Revisão & Psychopathology & 2018 \\
\hline 2 & $\begin{array}{l}\text { Kaess, M; } \\
\text { Parzer, p; } \\
\text { Koenig, j; } \\
\text { Resch, f; } \\
\text { Brunner, r. }\end{array}$ & $\begin{array}{c}\text { Dual-task } \\
\text { performance under } \\
\text { acute stress in } \\
\text { female adolescents } \\
\text { with borderline } \\
\text { personality disorder }\end{array}$ & BVS/Lilacs & $\begin{array}{l}\text { Ensaio } \\
\text { Clínico }\end{array}$ & $\begin{array}{c}\text { European Child } \\
\text { \& Adolescent } \\
\text { Psychiatry }\end{array}$ & 2016 \\
\hline 3 & $\begin{array}{l}\text { Kimmel CL; } \\
\text { Alhassoon } \\
\text { OM; } \\
\text { Wollman } \\
\text { SC; Stern } \\
\text { MJ; Perez- } \\
\text { Figueroa A; } \\
\text { Hall MG; }\end{array}$ & $\begin{array}{l}\text { Age-related parieto- } \\
\text { occipital and other } \\
\text { gray matter changes } \\
\text { in borderline } \\
\text { personality disorder: } \\
\text { A meta-analysis of } \\
\text { cortical and } \\
\text { subcortical } \\
\text { structures }\end{array}$ & pubMED/Medline & Revisao & $\begin{array}{c}\text { Psychiatry } \\
\text { Research: } \\
\text { Neuroimaging }\end{array}$ & 2016 \\
\hline
\end{tabular}




\begin{tabular}{|c|c|c|c|c|c|c|}
\hline & $\begin{array}{l}\text { Rompogren } \\
\text { J \& Radua J. }\end{array}$ & & & & & \\
\hline 4 & $\begin{array}{c}\text { Krause-Utz } \\
\text { A; Winter } \\
\text { D; } \\
\text { Niedtfeld I; } \\
\text { Schmahl C. }\end{array}$ & $\begin{array}{c}\text { The latest } \\
\text { neuroimaging } \\
\text { findings in } \\
\text { borderline } \\
\text { personality disorder. }\end{array}$ & pubMED/Medline & Revisão & $\begin{array}{l}\text { Current } \\
\text { Psychiatry } \\
\text { Reports }\end{array}$ & 2014 \\
\hline 5 & $\begin{array}{c}\text { Krause-Utz } \\
\text { AD; Elzinga } \\
\text { BM. }\end{array}$ & $\begin{array}{l}\text { [Neural mechanisms } \\
\text { of dissociation: } \\
\text { implication for } \\
\text { borderline } \\
\text { personality } \\
\text { disorder]. }\end{array}$ & pubMED/Medline & Revisao & $\begin{array}{c}\text { Tijdschrift voor } \\
\text { psychiatrie }\end{array}$ & 2019 \\
\hline 6 & $\begin{array}{c}\text { Lamers A; } \\
\text { Toepper M; } \\
\text { Fernando } \\
\text { SC; } \\
\text { Schlosser N; } \\
\text { Bauer E; } \\
\text { Woermann } \\
\text { F; Driessen } \\
\text { M \& Beblo } \\
\text { T. }\end{array}$ & $\begin{array}{l}\text { Nonacceptance of } \\
\text { negative emotions in } \\
\text { women with } \\
\text { borderline } \\
\text { personality disorder: } \\
\text { association with } \\
\text { neuroactivity of the } \\
\text { dorsal striatum }\end{array}$ & pubMED/Medline & $\begin{array}{l}\text { Ensaio } \\
\text { Clínico }\end{array}$ & $\begin{array}{c}\text { Journal of } \\
\text { Psychiatry \& } \\
\text { Neuroscience }\end{array}$ & 2019 \\
\hline 7 & $\begin{array}{l}\text { O'Neill A \& } \\
\text { Frodl T. }\end{array}$ & $\begin{array}{l}\text { Brain structure and } \\
\text { function in } \\
\text { borderline } \\
\text { personality disorder. }\end{array}$ & pubMED/Medline & Revisão & $\begin{array}{c}\text { Brain Structure } \\
\text { and Function }\end{array}$ & 2012 \\
\hline 8 & $\begin{array}{c}\text { Perez- } \\
\text { Rodriguez } \\
\text { MM; } \\
\text { Bulbena- } \\
\text { Cabré A; } \\
\text { Bassir Nia } \\
\text { A; Zipursky } \\
\text { G; } \\
\text { Goodman } \\
\text { M \& New } \\
\text { AS. }\end{array}$ & $\begin{array}{c}\text { The Neurobiology } \\
\text { of Borderline } \\
\text { Personality } \\
\text { Disorder. }\end{array}$ & pubMED/Medline & Revisão & $\begin{array}{c}\text { Psychiatric } \\
\text { Clinics of North } \\
\text { America }\end{array}$ & 2018 \\
\hline
\end{tabular}




\begin{tabular}{|c|c|c|c|c|c|c|}
\hline 9 & $\begin{array}{c}\text { Rossi, R., } \\
\text { Lanfredi, } \\
\text { M., Pievani, } \\
\text { M., } \\
\text { Boccardi, } \\
\text { M., Rasser, } \\
\text { P. E., } \\
\text { Thompson, } \\
\text { P. M., } \\
\text { Cavedo, E., } \\
\text { Cotelli, M., } \\
\text { Rosini, S., } \\
\text { Beneduce, } \\
\text { R., Bignotti, } \\
\text { S., Magni, } \\
\text { L. R., } \\
\text { Rillosi, L., } \\
\text { Magnaldi, } \\
\text { S., Cobelli, } \\
\text { M., Rossi, } \\
\text { G., \& } \\
\text { Frisoni, G. } \\
\text { B }\end{array}$ & $\begin{array}{l}\text { Abnormalities in } \\
\text { cortical gray matter } \\
\text { density in borderline } \\
\text { personality disorder }\end{array}$ & pubMED/Medline & $\begin{array}{l}\text { Ensaio } \\
\text { Clínico }\end{array}$ & $\begin{array}{l}\text { European } \\
\text { Psychiatry }\end{array}$ & 2015 \\
\hline IO & $\begin{array}{l}\text { Ruocco AC } \\
\text { \& Bahl N. }\end{array}$ & $\begin{array}{l}\text { Material-specific } \\
\text { discrepancies in } \\
\text { verbal and visual } \\
\text { episodic memory in } \\
\text { borderline } \\
\text { personality disorder. }\end{array}$ & pubMED/Medline & $\begin{array}{l}\text { Ensaio } \\
\text { Clínico }\end{array}$ & $\begin{array}{l}\text { Psychiatry } \\
\text { Research }\end{array}$ & 2014 \\
\hline II & $\begin{array}{l}\text { Ruocco AC, } \\
\text { Carcone D. }\end{array}$ & $\begin{array}{c}\text { A Neurobiological } \\
\text { Model of Borderline } \\
\text { Personality } \\
\text { Disorder: } \\
\text { Systematic and } \\
\text { Integrative Review. }\end{array}$ & pubMED/Medline & Revisão & $\begin{array}{c}\text { Harvard Review } \\
\text { of Psychiatry }\end{array}$ & 2016 \\
\hline 12 & $\begin{array}{l}\text { Scherpiet S, } \\
\text { Herwig U, } \\
\text { Opialla S, } \\
\text { Scheerer H, } \\
\text { Habermeyer } \\
\text { V, Jäncke L, } \\
\text { Brühl AB. }\end{array}$ & $\begin{array}{l}\text { Reduced neural } \\
\text { differentiation } \\
\text { between self- } \\
\text { referential cognitive } \\
\text { and emotional } \\
\text { processes in women } \\
\text { with borderline } \\
\text { personality disorder. }\end{array}$ & pubMED/Medline & $\begin{array}{l}\text { Ensaio } \\
\text { Clínico }\end{array}$ & $\begin{array}{l}\text { Psychiatry } \\
\text { Research }\end{array}$ & 2015 \\
\hline
\end{tabular}




\begin{tabular}{|c|c|c|c|c|c|c|}
\hline 13 & $\begin{array}{c}\text { Schmahl, } \\
\text { C., } \\
\text { Herpertz, S. } \\
\text { C., Bertsch, } \\
\text { K., Ende, } \\
\text { G., Flor, H., } \\
\text { Kirsch, P., } \\
\text { Lis, S., } \\
\text { Meyer- } \\
\text { Lindenberg, } \\
\text { A., } \\
\text { Rietschel, } \\
\text { M., } \\
\text { Schneider, } \\
\text { M., } \\
\text { Spanagel, } \\
\text { R., Treede, } \\
\text { R. D., \& } \\
\text { Bohus, M }\end{array}$ & $\begin{array}{c}\text { Mechanisms of } \\
\text { disturbed emotion } \\
\text { processing and social } \\
\text { interaction in } \\
\text { borderline } \\
\text { personality disorder: } \\
\text { state of knowledge } \\
\text { and research agenda } \\
\text { of the German } \\
\text { Clinical Research } \\
\text { Unit }\end{array}$ & pubMED/Medline & Revisão & $\begin{array}{c}\text { Borderline } \\
\text { Personality } \\
\text { Disorder and } \\
\text { Emotion } \\
\text { Dysregulation }\end{array}$ & 2014 \\
\hline I4 & $\begin{array}{c}\text { Schmitz M, } \\
\text { Müller LE, } \\
\text { Schulz A, } \\
\text { Kleindienst } \\
\text { N, Herpertz } \\
\text { SC, Bertsch } \\
\text { K. }\end{array}$ & $\begin{array}{l}\text { Heart and brain: } \\
\text { Cortical } \\
\text { representation of } \\
\text { cardiac signals is } \\
\text { disturbed in } \\
\text { borderline } \\
\text { personality disorder, } \\
\text { but unaffected by } \\
\text { oxytocin } \\
\text { administration. }\end{array}$ & pubMED/Medline & $\begin{array}{l}\text { Ensaio } \\
\text { clínico }\end{array}$ & $\begin{array}{c}\text { Journal of } \\
\text { Affective } \\
\text { Disorders }\end{array}$ & 2020 \\
\hline I5 & $\begin{array}{c}\text { Schulze, L., } \\
\text { Grove, M., } \\
\text { Tamm, S. et } \\
\text { al. }\end{array}$ & $\begin{array}{c}\text { Effects of } \\
\text { transcranial direct } \\
\text { current stimulation } \\
\text { on the cognitive } \\
\text { control of negative } \\
\text { stimuli in borderline } \\
\text { personality disorder. }\end{array}$ & pubMED/Medline & $\begin{array}{l}\text { Ensaio } \\
\text { Clínico }\end{array}$ & $\begin{array}{c}\text { Scientific } \\
\text { Reports }\end{array}$ & 2019 \\
\hline I6 & $\begin{array}{c}\text { Sebastian A, } \\
\text { Jung P, } \\
\text { Krause-Utz } \\
\text { A, Lieb K, } \\
\text { Schmahl C, } \\
\text { Tüscher O. }\end{array}$ & $\begin{array}{l}\text { Frontal dysfunctions } \\
\text { of impulse control - } \\
\text { a systematic review } \\
\text { in borderline } \\
\text { personality disorder } \\
\text { and attention- } \\
\text { deficit/hyperactivity } \\
\text { disorder. }\end{array}$ & pubMED/Medline & Revisão & $\begin{array}{c}\text { Frontiers in } \\
\text { Human } \\
\text { Neuroscience }\end{array}$ & 2014 \\
\hline 17 & $\begin{array}{c}\text { Vai B; } \\
\text { Cazzetta S; } \\
\text { Scalisi R; } \\
\text { Donati A; } \\
\text { Bechi M; } \\
\text { Poletti S; } \\
\text { Sforzini L; }\end{array}$ & $\begin{array}{l}\text { Neuropsychological } \\
\text { deficits correlate } \\
\text { with symptoms } \\
\text { severity and cortical } \\
\text { thickness in } \\
\text { Borderline } \\
\text { Personality Disorder }\end{array}$ & pubMED/Medline & $\begin{array}{l}\text { Ensaio } \\
\text { Clínico }\end{array}$ & $\begin{array}{l}\text { Journal of } \\
\text { Affective } \\
\text { Disorders }\end{array}$ & 2021 \\
\hline
\end{tabular}




\begin{tabular}{|c|c|c|c|c|c|c|}
\hline & $\begin{array}{l}\text { Visintini R; } \\
\text { Maffei C \& } \\
\text { Benedetti F. }\end{array}$ & & & & & \\
\hline 18 & $\begin{array}{l}\text { Schreiner } \\
\text { M; Klimes- } \\
\text { Dougan B; } \\
\text { Mueller BA; } \\
\text { Nelson KJ; } \\
\text { Lim KO; } \\
\text { Cullen KR. }\end{array}$ & $\begin{array}{c}\text { Neurocircuitry } \\
\text { associated with } \\
\text { symptom } \\
\text { dimensions at } \\
\text { baseline and with } \\
\text { change in borderline } \\
\text { personality disorder. }\end{array}$ & pubMED/Medline & $\begin{array}{l}\text { Ensaio } \\
\text { clínico }\end{array}$ & $\begin{array}{c}\text { Psychiatry } \\
\text { Research: } \\
\text { Neuroimaging }\end{array}$ & 2019 \\
\hline 19 & $\begin{array}{c}\text { Zaehringer } \\
\text { J; Ende G; } \\
\text { Santangelo } \\
\text { P; } \\
\text { Kleindienst } \\
\text { N; Ruf M; } \\
\text { Bertsch K; } \\
\text { Bohus M; } \\
\text { Schmahl C; } \\
\text { Paret C. }\end{array}$ & $\begin{array}{l}\text { Improved emotion } \\
\text { regulation after } \\
\text { neurofeedback: A } \\
\text { single-arm trial in } \\
\text { patients with } \\
\text { borderline } \\
\text { personality disorder. }\end{array}$ & pubMED/Medline & $\begin{array}{l}\text { Ensaio } \\
\text { clínico }\end{array}$ & $\begin{array}{c}\text { NeuroImage: } \\
\text { Clinical }\end{array}$ & 2019 \\
\hline 20 & $\begin{array}{c}\text { Zegarra- } \\
\text { Valdivia, J; } \\
\text { Vilca, B. }\end{array}$ & $\begin{array}{l}\text { Social cognition and } \\
\text { executive function } \\
\text { in borderline } \\
\text { personality disorder: } \\
\text { evidence of altered } \\
\text { cognitive processes }\end{array}$ & sciELO/Medline & $\begin{array}{l}\text { Ensaio } \\
\text { Clínico }\end{array}$ & Salud Mental & 2019 \\
\hline
\end{tabular}

Ao observar a quantidade de produções no período de 2010 a 2021, constatou-se uma maior concentração de artigos publicados em 2019 (30\%), e logo em seguida, uma disposição com uma menor disparidade entre 2018 (I0\%), 2016 (15\%) e 2015 (10\%). Por fim, os anos de 2012, 2020 e 202I concentram 5\% dos artigos cada um. Sendo assim, o ano de 2019 foi o período de maior desenvolvimento de pesquisas acerca da temática em questão.

No que concerne ao enfoque das publicações, foram identificadas no transtorno de personalidade borderline alterações a nível cognitivo, assim como em estrutura e função de determinadas regiões do cérebro desde a região límbica a alterações no córtex cerebral como um todo. Mais especificamente, na região límbica reconheceram-se alterações com maior ênfase na região da amigdala, do hipocampo e hipotálamo. Por fim, também observou-se a 
existência de alterações que transcendiam o espaço cerebral, bem como a menção sobre fatores genéticos.

Gráfico I - Distribuição da quantidade de artigos anualmente, no período de 2010 à 202I.

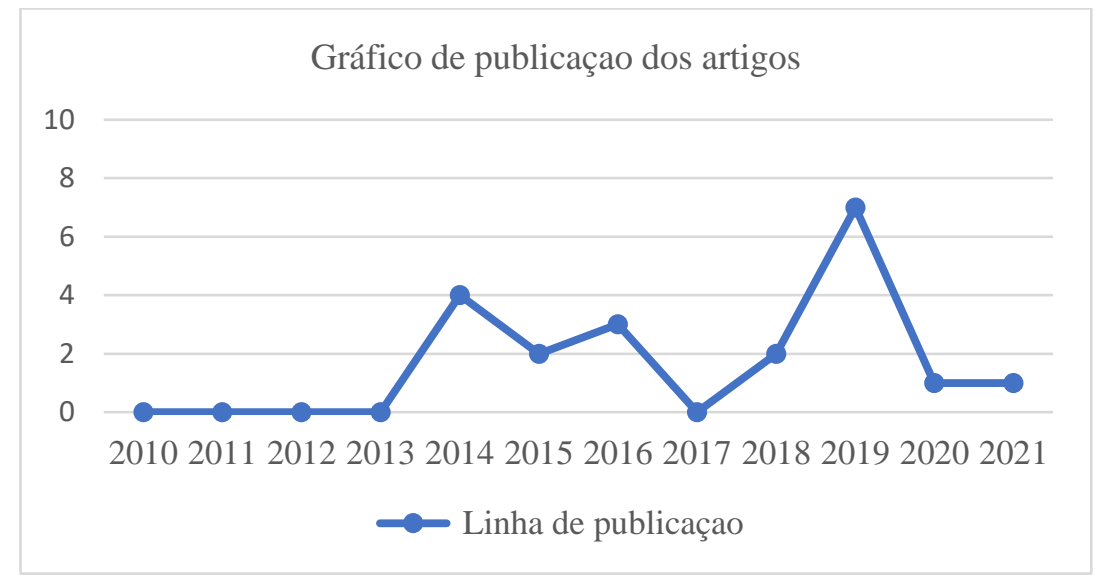

Fonte: Os autores (2021).

Assim, com o intuito de apresentar de maneira pormenorizada os dados encontrados, estes foram expostos nos Quadros 2, 3, 4, 5, levando em consideração a ordem de enumeração dos artigos apresentados no Quadro I. Logo, cada item possui ao seu lado a numeração dos artigos correspondentes.

As alterações cognitivas mais recorrentes entre os trabalhos coletados (Quadro 2) são: menor funcionamento adaptativo (20\%); alterações na memória (I0\%); alterações na atenção (IO\%); alterações nas tomadas de decisão (I0\%); alterações nos processos autorreferenciais (Іо\%); alterações nas respostas ao estresse (I0\%); e alterações no processamento emocional (I0\%).

Quadro 2. Alterações cognitivas de acordo a enumeração do Quadro I.

\begin{tabular}{|c|c|c|}
\hline ITEM & ALTERAÇÕES COGNITIVAS & ARTIGOS \\
\hline I & Menor funcionamento adaptativo & {$[2],[4],[\mathrm{Io}],[20]$} \\
\hline 2 & Alterações na memória & {$[5],[\mathrm{Io}]$} \\
\hline 3 & Alterações nas respostas ao estresse & {$[2],[5]$,} \\
\hline 4 & Alterações no processamento emocional & {$[5],[20]$} \\
\hline 5 & Alterações na atenção & {$[5],[\mathrm{Io}]$} \\
\hline 6 & Alterações nos processos autorreferenciais & {$[4],[5]$,} \\
\hline
\end{tabular}




\begin{tabular}{|c|c|c|}
\hline 7 & Alterações nas tomadas de decisão & [10], [16] \\
\hline 8 & Hipersensibilidade à ameaças & {$[\mathrm{I}]$} \\
\hline 9 & Alterações no reconhecimento de estímulos auditivos & [2] \\
\hline Io & Alterações no reconhecimento de estímulos visuais & [2] \\
\hline II & Alterações no tempo de reação dos estímulos auditivos & [2] \\
\hline 12 & Alterações no tempo de reação dos estímulos visuais & [2] \\
\hline 13 & Alterações na velocidade psicomotora, & [10] \\
\hline 14 & Alterações nas habilidades visuoespaciais & [10] \\
\hline 15 & Alterações na aprendizagem & [10] \\
\hline
\end{tabular}

*O símbolo “[ ]” acompanhado de uma enumeração no eixo artigos faz menção a enumeração do artigo no Quadro I e a respectiva ordem apresentada.

Fonte: Os autores (2021).

Em relação às alterações na região límbica (Quadro 3), os artigos ressaltavam com maior frequência alterações na amígdala, hipocampo e hipotálamo. Sendo assim, os achados foram subdividos entre essas três esferas. Entre as alterações da amígdala mais destacadas estão: aumento das ativações na amígdala (35\%); redução da ativação da amígdala (25\%) e a diminuição do volume da amígdala (15\%). No que se refere ao hipocampo, os artigos fizeram menção a: diminuição do volume do hipocampo (I5\%); baixo volume da massa cinzenta do hipocampo (5\%) e o aumento da ativação no hipocampo esquerdo na exposição a estímulos negativos (5\%). Por fim, na região do hipotálamo há menção apenas ao aumento de volume neste (5\%). Destaca-se aqui que, do total dos 20 artigos coletados (Quadro I), 70\% deles apresentavam alterações na amígdala $(\mathrm{n}=12)$.

Quadro 3. Alterações na região límbica de acordo a enumeração do Quadro I.

\begin{tabular}{|c|c|c|}
\hline \multicolumn{2}{|c|}{ AMÍGDALA } \\
\hline ITEM & ALTERAÇÕES & ARTIGOS \\
\hline I & Aumento das ativações na amígdala & {$[\mathrm{I}],[4],[7],[\mathrm{II}],[\mathrm{I} 3]$,} \\
& & {$[\mathrm{I} 6]$} \\
\hline 2 & Redução da ativação da amígdala & {$[5],[6],[\mathrm{II}],[\mathrm{I} 2],[\mathrm{I} 6]$} \\
\hline 3 & Diminuição do volume da amígdala & {$[7],[\mathrm{II}],[\mathrm{I} 3]$} \\
\hline 4 & Redução do volume da massa cinzenta com o passar da idade & {$[3]$} \\
\hline
\end{tabular}




\begin{tabular}{|c|c|c|}
\hline 5 & Aumento do volume da amígdala & [9] \\
\hline 6 & Mudanças na conectividade funcional da amígdala; & {$[5]$} \\
\hline 7 & Baixa creatina & [II] \\
\hline 8 & Redução de $\mathrm{N}$-acetil aspartato & {$[13]$} \\
\hline \multicolumn{3}{|c|}{ HIPOCAMPO } \\
\hline ITEM & ALTERAÇÕES & ARTIGOS \\
\hline I & Diminuição do volume do hipocampo & {$[7],[\mathrm{II}],[\mathrm{I} 3]$} \\
\hline 2 & Baixo volume da massa cinzenta do hipocampo & {$[3]$} \\
\hline 3 & $\begin{array}{l}\text { Aumento da ativação no hipocampo esquerdo na exposição a estímulos } \\
\text { negativos }\end{array}$ & {$[6]$} \\
\hline \multicolumn{3}{|c|}{ HIPOTÁLAMO } \\
\hline ITEM & ALTERAÇÕES & ARTIGOS \\
\hline I & Aumento de volume no hipotálamo & [13] \\
\hline
\end{tabular}

*O símbolo "[ ]" acompanhado de uma enumeração no eixo artigos faz menção a enumeração do artigo no Quadro i e a respectiva ordem apresentada.

Fonte: Os autores (2021).

Ao observar o Quadro 4, evidenciam-se outras alterações presentes na região cerebral, sobretudo aquelas que não fazem menção à região límbica, haja vista que as alterações dessa região já foram expostas. Posto isso, as outras alterações mais encontradas fazem menção a: alterações nas regiões de controle pré-frontal (20\%); maior concentração de $\mathrm{N}$-acetilasparto e glutamato (I5\%); alteração na região orbitofrontal, dorso-medial e dorsolateral pre-frontal (Io\%) e ao aumento da ativação no córtex cingulado posterior esquerdo (Io\%).

Quadro 4. Outras alterações nas regiões do cérebro no geral.

\begin{tabular}{|c|c|c|}
\hline ITEM & ALTERAÇÕES & ARTIGOS \\
\hline I & Alterações nas regiões de controle pré-frontal & $\begin{array}{l}{[4],[13],} \\
{[15],[16],}\end{array}$ \\
\hline 2 & Maior concentração de $\mathrm{N}$-acetilasparto e glutamato & {$[7],[\mathrm{II}],[\mathrm{I} 3]$} \\
\hline 3 & Alteração na região orbitofrontal, dorso-medial e dorsolateral pre-frontal & {$[2],[16]$} \\
\hline 4 & Aumento da ativação no córtex cingulado posterior esquerdo & {$[6][12]$} \\
\hline 5 & Alterações da massa cinzenta em áreas do cérebro externas a região límbica & [3] \\
\hline
\end{tabular}




\begin{tabular}{|c|c|c|}
\hline 6 & Ativação diminuída no córtex cingulado médio esquerdo & {$[4]$} \\
\hline 7 & Ativação nas regiões frontoestriatais do cérebro (giro frontal superior \\
esquerdo, caudado direito & {$[6]$} \\
\hline 8 & $\begin{array}{c}\text { Diminuição na densidade em áreas especificas no córtex. } \\
\text { Maior densidade no hemisfério direito. }\end{array}$ & {$[9]$} \\
\hline 9 & Menor volume nos lobos frontais & {$[9]$} \\
\hline IO & Aumento de volume no putame. & {$[\mathrm{I} 3]$} \\
\hline II & hipoatividade do córtex adrenal & {$[\mathrm{I} 6]$} \\
\hline I2 & Diminuição na ativação no giro frontal inferior esquerdo com inibição de \\
\end{tabular}

*O símbolo "[ ]" acompanhado de uma enumeração no eixo artigos faz menção a enumeração do artigo no Quadro I e a respectiva ordem apresentada.

Fonte: Os autores (2021).

Diante da amplitude de alterações ou outros fatores que podem vir a ocorrer no TPB que não correspondem às já elucidadas, o Quadro 5 apresenta algumas alterações menos específicas. Dentre estas, a alteração na frequência cardíaca diante de estressores (Io\%) é a que mais se destaca, não obstante a menção à fatores genéticos para acúmulo de $\mathrm{N}$ Acetilasparto chamem demasiada atenção.

Quadro 5. Algumas alterações menos especificas

\begin{tabular}{|c|c|c|}
\hline ITEM & ALTERAÇÕES & ARTIGOS \\
\hline I & Alteração na frequência cardíaca diante de estressores & {$[2],[\mathrm{I} 4]$} \\
\hline 2 & Baixa ocitocina sérica & {$[\mathrm{II}]$} \\
\hline 3 & Maior crescimento endotelial vascular & {$[\mathrm{II}]$} \\
\hline 4 & Níveis elevados de ácidos graxos & {$[\mathrm{II}]$} \\
\hline 5 & Fator genético para acúmulo de N-acetilasparto & {$[\mathrm{I} 3]$} \\
\hline 6 & Redução de N-acetil aspartato & {$[7]$} \\
\hline 7 & Herdabilidade & \\
\hline
\end{tabular}

Fonte: Os autores (2021).

\section{Discussão}

O transtorno de personalidade borderline (TPB) é uma doença mental presente em média de I,6\% da população em geral (Chapman, Jamil \& Fleisher, 2021), comumente 
caracterizado por sua instabilidade na regulação do afeto, controle de impulso, relações interpessoais e autopercepção (APA, 2014).

Uma vez que o TPB é um transtorno clinicamente heterogêneo, a importância de reconhecer a especificidade das características e alterações neuropsicológicas se alinham para um olhar mais atento sobre a patologia. Para fazer avançar essas iniciativas, há de se ter em consciência a existência de alterações em alguns mecanismos cognitivos e biológicos.

\section{Alterações cognitivas}

Com demasiada regularidade, o TPB pode proporcionar um menor funcionamento adaptativo; ou seja, os indivíduos diagnosticados com TPB podem apresentar prejuízos significativos nas habilidades sociais, práticas e conceituais que agem na manutenção da adaptação ao ambiente.

De acordo com Krause-Utz et al. (2014), em um estudo de revisão com foco nas últimas pesquisas de neuroimagem em TPB com ênfase em estudos de ressonância magnética funcionais e de estruturas, o menor funcionamento adaptativo é cercado por um

conjunto de variáveis. Assim, o menor funcionamento adaptativo teria uma correlação com a desregulação emocional e a impulsividade que, por vez, proporcionariam prejuízos na formação das relações interpessoais intimamente relacionadas com ele. Além disso, essas relações estariam associadas a alterações cerebrais e frontolímbicas. Corroborando com isso, em um ensaio clínico com amostra de 30 adolescentes com TPB e 34 sem TPB, os pacientes com TPB destacaram um menor funcionamento adaptativo em comparação aos que não possuíam o TPB, de acordo com os critérios do DSM (Kaess et al., 2016).

Além disso, em um outro estudo no qual foram avaliadas 20 mulheres com TPB em internação ambulatorial e 20 mulheres sem TPB, o menor funcionamento adaptativo estaria condicionado à mentalização, ou seja, teria sua relação com a maior vigilância quanto às emoções, intenções e pensamentos negativos. Consequentemente, reduziria as habilidades de integração dos estímulos sociais com interpretações não adequadas deste contexto (Zegarra-Valdivia \& Vilca, 2019).

Dentre as outras alterações cognitivas que o TPB ocasiona, a atenção e memória se destacam pela amplitude dessas alterações. No TPB, os processos cognitivos da atenção e memória se apresentam significativamente afetados. De acordo com Ruocco \& Bahl (2014), 
os problemas de atenção eventualmente podem estar associados a uma redução da consciência no momento presente. No entanto, Krause \& Elzinga (2019) destaca que esses prejuízos na atenção - neste caso, maior distração - acontecem na exposição a informações emocionais e, por consequência, afetaria o domínio da memória. Assim, conclui-se que os pacientes com TPB podem apresentar dificuldades nas tarefas diárias.

Ainda sobre o domínio da memória, em um estudo com 24 pacientes diagnosticados com TPB, a partir da realização de testes de memória episódica co-normatizados (Hopkins Verbal Learning Test e Brief Visuopatial Memory Test), foi possível concluir que os pacientes com TPB apresentavam uma maior probabilidade - três vezes mais - de mostrar discrepâncias em sua recordação de materiais verbais e visuais da memória (Ruocco \& Bahl, 2014).

Destaca-se em especial que na dissociação às vezes presente no $\mathrm{TPB}$, os pacientes apresentam um maior prejuízo nos domínios cognitivos, inclusive na memória e atenção (Krause \& Elzinga, 2019).

Outro prejuízo relacionado ao TPB é uma maior dificuldade no processamento emocional por partes de seus portadores (Krause \& Elzinga, 2019; Zegarra-Valdivia \& Vilca, 2019). Assim, torna-se possível correlacionar com os achados referentes a respostas ao estresse. Dito isso, se os indivíduos com TPB apresentam prejuízo no processamento emocional, não seria novidade, tal como apresenta Kaess et al. (2016), uma maior sensibilidade ao estresse; logo, um maior nível de irritabilidade. Do ponto de vista cerebral, esses prejuízos teriam uma íntima relação com a amígdala e o córtex cingulado anterior, áreas envolvidas nas respostas ao estresse e no processamento emocional (Krause \& Elzinga, 2019).

Para além das alterações supracitadas, indivíduos com o TPB também podem vir a apresentar alterações nos processos autorreferenciais e de tomada de decisão (Ruocco \& Bahl, 2014).

A partir das alterações cognitivas, torna-se possível inferir que elas se correlacionam. Sendo assim, determinadas alterações em um domínio cognitivo podem alterar outros, como já demonstrado no caso da atenção e memória, mas também envolvendo as outras alterações, como também é possível visualizar no caso do menor funcionamento adaptativo e o 
processamento emocional, assim como envolvendo as respostas ao estresse e aos processos autorreferenciais.

\section{Alterações límbicas}

Com frequência, os mais diferentes estudos destacam a evidente importância da região límbica e suas alterações no que se refere ao transtorno de personalidade borderline desde alterações na amígdala a alterações no hipocampo e hipotálamo.

Responsável por nossas respostas emocionais e pelo seu papel nas características emocionais das nossas memórias, a amígdala no TPB apresenta alterações significativas na sua estrutura e função.

Conforme demonstra Bertsch, Hillmann \& Herpertz (2018), uma maior ativação da região da amígdala está relacionada a estímulos emocionalmente negativos, bem como aqueles estímulos que se configuram como ameaça. Em dois estudos de revisão realizados por Krause-Utz et al. (2014) e Sebastian et al. (2014), foi possível concluir a partir da coleta que há uma maior probabilidade que pacientes com TPB possam apresentar tempos de reação a estímulos mais longos devido à alta ativação da amígdala e da ínsula durante a distração emocional. Em alguns casos, destacam-se respostas atrasadas e ativação aprimorada da amígdala esquerda associadas às imagens carregadas emocionalmente (Sebastian et al., 2014)

Em um outro estudo de revisão acerca da estrutura e função do cérebro no transtorno de personalidade limítrofe, também é demonstrado que uma maior ativação da amigdala em pacientes com TPB ocorre quando confrontado com estímulos associados à emoção (O'neill \& Frodl, 2012). Igualmente, em um outro trabalho, os autores fazem menção a certos centros cerebrais que estão conectados com a região da amígdala e agem durante a percepção de expressões faciais negativas por parte dos pacientes com TPB (Ruocco \& Carcone, 2016). Em complemento, ainda de acordo com o autor, mesmo em ocasiões induzidas para o controle dos estímulos associados à emoção, sobretudo negativos, pacientes com TPB, por vez, não ativam todas as regiões neurais que fornecem a manutenção do controle negativo (Ruocco \& Carcone, 2016). 
Essas considerações, de alguma maneira, permitem o trabalho de associação com aqueles achados há pouco mencionados acerca do processamento emocional e, em especial, sobre o funcionamento adaptativo.

Ademais, alterações na memória de trabalho e na atenção associadas a distrações mostraram-se relacionadas ao aumento das ativações da amígdala (Schmahl et al., 20I4; Ruocco \& Carcone, 2016)

Em contrapartida, contrariando as expectativas, alguns estudos também apresentaram atividade reduzida da amígdala em pacientes diagnosticados com TPB (Scherpiet et al., 2015; Krause \& Elzinga, 2019; Lamers et al., 2019).

De acordo com Ruocco \& Carcone (2016), a atividade reduzida da amígdala acontece especificamente na amígdala direita. Em acréscimo a isso, em um estudo realizado com 40 mulheres, sendo 20 com TPB e 20 sem TPB, é destacada uma menor ativação nas áreas que circundam a região límbica, assim como na própria, destacando uma menor ativação da amígdala e do hipocampo quando confrontado com estímulos classificados como negativos (Lamers et al., 2019); o que de alguma forma entra em questão com os achados mencionados anteriormente a esse respeito.

Além disso, uma menor atividade da amígdala também é destacada em pacientes com TPB no processo de dissociação, sobretudo enquanto se deparavam com imagens de expressão emocional significativa (Krause \& Elzinga, 2019). Ainda sobre o processo de dissociação no TPB e sua relação com a amígdala enquanto função, destacam-se algumas mudanças na conectividade funcional da amígdala - em especial na distração - em que apresentam um movimento de correlação entre as atividades da amígdala e outras regiões do cérebro que correspondem à regulação da emoção (Krause \& Elzinga, 2019).

No que se refere a outras alterações enquanto função na amígdala, há menção a uma redução de $\mathrm{N}$-acetil aspartato (Schmahl et al., 2014) e baixa creatina (Ruocco \& Carcone, 2016). Segundo Schmahl et al. (2014), em alguns casos, pacientes com TPB apresentam redução de N-Acetil Aspartato; o que sugere o questionamento sobre lesão cerebral associada ao TPB, haja vista que o $\mathrm{N}$-Acetil Aspartato é entendido como um marcador neuronal que apresenta redução em casos de lesão cerebral. Já a baixa creatina, intimamente associada ao metabolismo energético, em que suas alterações em concentração, por vez, aparecem como 
resultado de morte celular ou trauma cerebral, pode ser um guia para o entendimento das concentrações de metabólitos em pacientes com TPB (Ruocco \& Carcone, 2016).

As alterações funcionais do hipocampo fazem menção a uma maior ativação da região. Segundo Lamers et al. (2019), os indivíduos com TPB também apresentam maior ativação na região do hipocampo em comparação a pacientes sem o transtorno, em especial ao responder situações negativas em contraste com situações neutras.

Do ponto de vista da estrutura, os achados fazem menção à redução do volume em algumas partes da região límbica, em especial na amígdala e no hipocampo (O'neill \& Frodl, 2012; Schmahl et al., 2014; Ruocco \& Carcone, 2016).

No estudo de revisão realizado por O'Neill \& Frodl (2012), a partir de um conjunto de ensaios clínicos, é enfatizada a redução da amígdala em pacientes portadores do TPB. Nos casos em questão, independentemente da idade e do sexo, os achados corroboravam e apresentavam resultados deveras semelhante. Ou seja, em todos os casos que os pacientes com TPB foram comparados a pacientes não portadores do TPB, as diferenças foram significantes e apontavam para o fato em questão. Além disso, dentre os outros achados coletados nesta revisão, um deles chama a atenção para a possibilidade de essas mudanças na amígdala apontarem para diferentes características do transtorno.

Segundo Ruocco \& Carcone (2016), em um estudo de revisão consideravelmente mais recente acerca das características neurobiológicas do TPB, permanece o fato de a redução do volume na amígdala ser um fator preponderante nos casos de TPB. Contudo, no que transcende a essa questão, dentre os achados deste estudo, uma demasiada atenção recai sobre a afirmativa da maior prevalência de estudos que fazem menção para um menor volume da amígdala estar relacionado a traumas na infância. Em acréscimo, destaca-se o menor volume na amígdala bilateral.

Embora contrastante com a literatura e os achados aqui já mencionados, o aumento do volume da amígdala em pacientes portadores do TPB também é presente (Rossi et al., 2015). No entanto, pouco é mencionado acerca dos desdobramentos dessa afirmativa, e assim se mostra inconsistente diante da literatura exposta.

No que se refere ao hipocampo, segundo O'Neill \& Frodl (2012) e Schmahl et al. (2014), a redução do volume do hipocampo aparece com maior regularidade nas pesquisas a esse respeito. Tais alterações no volume aparecem correlacionadas pela história de abuso e a 
duração do abuso na infância (O'neill \& Frodl, 2012). Ainda de acordo com o autor, a diminuição do volume pode levar a prejuízos neurocognitivos, sintomas dissociativos, distorções perceptivas e instabilidade de identidade, sintomas estes já comumente encontrados em pacientes com TPB. Além disso, curiosamente, embora pacientes com TPB apresentem redução do volume do hipocampo, no hipotálamo podem apresentar aumento do volume (Schmahl et al., 2014),

Ainda sobre as alterações límbicas a nível de estrutura, em uma pesquisa realizada por Kimmel et al. (2016) acerca das alterações parieto-occipitais relacionadas à idade e outras alterações da massa cinzenta no TPB, sobretudo a partir de uma meta-análise das estruturas corticais e subcorticais, redução no volume da massa cinzenta na região da amígdala é frequentemente encontrada em pacientes com TPB. No entanto, de acordo com o que foi encontrado pelos autores, a diminuição do volume da massa cinzenta está relacionada à idade, em que, com o passar dos anos, há uma maior propensão para essa diminuição.

Tal como na amígdala, pacientes com TPB aparentemente também apresentam um menor volume da massa cinzenta na região do hipocampo (O'neill \& Frodl, 20I2; Kimmel et al., 2016). Conforme o conjunto de resultados encontrados por Kimmel et al. (2016), pacientes com TPB apresentam redução da massa cinzenta no hipocampo esquerdo, assim como no giro frontal inferior direito pars opercularis e giro temporal médio direito. Além disso, o baixo volume da massa cinzenta do hipocampo apresenta-se associado a prejuízos na função da memória autobiográfica, agressão reativa e até mesmo nos sintomas dissociativos (Kimmel et al., 2016).

Alterações em outras regiões do cérebro externas à região límbica

Assim como na região límbica, outras regiões do cérebro em pacientes com o Transtorno de personalidade Borderline também apresentam algumas alterações importantes.

Segundo Krause-Utz et al. (2014), pacientes diagnosticados com TPB regularmente apresentam alterações no córtex pré-frontal. Em seu estudo de revisão, os resultados destacam que pacientes com TPB quando solicitados a afastar imagens negativas de seu pensamento, apresentam atividade reduzida no córtex pré-frontal ventrolateral. No entanto, 
conforme Sebastian et al. (2014), embora a presença de alterações no TPB manifeste-se constantemente através de uma combinação de diferentes regiões do cérebro, os prejuízos associados ao controle cognitivo de estímulos negativos são resultados da baixa atividade do córtex pré-frontal dorsolateral. Assim, por consequência, essas alterações nessa região do córtex pré-frontal direcionam para uma associação com a evidente deficiência dos pacientes borderline no processamento emocional. Portanto, esse achado sugere uma correspondência com as considerações feitas acerca do processamento emocional nas alterações cognitivas anteriormente.

Ainda segundo Krause-Utz et al. (2014), de maneira mais específica, não obstante pouco minuciosa, os pacientes com TPB também apresentam maior ativação no córtex préfrontal medial; logo, sugere-se que tal ativação possui correspondência com a sensibilidade a rejeição e, em especial, o medo de abandono. Além disso, alterações na região pré-frontal medial apresentam uma relação direta na desregulação emocional, bem como nas condições de inibição comportamental (Sebastian et al., 2014), dado que os processos associados às áreas do córtex pré-frontal medial têm sua relação com o controle e modulação da ativação emocional relacionadas com estratégias de respostas (Schmahl et al., 2015).

Com base em Kaess et al. (2016), déficit nas funções executivas superiores habilidades cognitivas essenciais para o controle dos pensamentos, emoções e ações refletem alterações negativas consideráveis no córtex pré-frontal orbitofrontal e dorsolateral. Além disso, em um estudo de investigação através de ressonância magnética funcional, com amostra de 19 pacientes do sexo feminino com TPB e 19 sem TPB comparados durante o processo de introspecção consciente, autorreflexão cognitiva e uma condição neutra, pacientes com TPB apresentaram maior ativação no córtex cingulado posterior esquerdo (Lamers et al., 2019). Igualmente, em um outro estudo realizado com 20 mulheres com TPB e 20 sem o transtorno, as pacientes diagnosticadas também apresentaram maior ativação no córtex cingulado posterior esquerdo, principalmente na região da substância branca (Scherpiet et al., 2015).

Além disso, pacientes com TPB também apresentam maior ativação nas regiões frontoestriatais do cérebro, em especial no giro frontal superior esquerdo e caudado (Lamers et al., 2019), haja vista que os circuitos frontoestriatais do cérebro podem ser compreendidos como vias neurais específicas que conectam as regiões do lobo frontal com os conhecidos 
núcleos da base - estriado - que têm por função mediar as funções cognitivas, comportamentais e motoras.

Segundo O'Neill \& Frodl (2012) e Ruocco \& Carcone (2016), em pacientes diagnosticados com borderline encontram-se um maior nível de concentração de $\mathrm{N}$ acetilasparto e glutamato, ambos extremamente implicados na regulação emocional e nos processos de controle cognitivo.

Por fim, pacientes com borderline aparentam apresentar diminuição na densidade em áreas específicas do córtex, mais especificamente nas áreas do giro pré-frontal temático e a junção temporoparietal e pré-cuneus, menor densidade de tecido no giro temporal superior bilateral e no giro frontal inferior e médio, assim como menor densidade nos córtexs cingulado do hemisfério esquerdo (Rossi et al., 2015). Enfim, de acordo com Ruocco \& Carcone (2016), em um dos resultados da sua revisão, mulheres com TPB demonstravam volumes menores dos lobos frontais.

Outras alterações menos específicas

Embora as alterações mencionadas nos tópicos anteriores possuam um demasiado destaque, por vez, outras alterações menos específicas associadas ao TPB também aparecem, dentre estas, segundo Kaess et al. (2016) e Schmitz et al. (2020), a alteração na frequência cardíaca, em certa medida, chama uma maior atenção. De acordo com ambos, a alteração na frequência cardíaca em pacientes com borderline é frequente - tanto para o aumento quanto para sua diminuição, embora seja mais frequente o aumento - e pode aparecer especialmente em situações de estresse elevado ou na exposição a contextos emocionais carregados. Além dessa alteração, Ruocco \& Carcone (2016) destacam: baixa ocitocina sérica; maior crescimento endotelial vascular; níveis elevados de ácidos graxos, no qual podem estar associados aos níveis de estresse, sensibilidade a dor, humor e cognição no TPB; fatores genéticos para o acúmulo de $\mathrm{N}$-acetil Aspartato e, principalmente, fatores hereditários para o desenvolvimento do TPB, em que, segundo O'Neill \& Frodl (2012), embora esses fatores ainda sejam imprecisos, alguns estudos sustentam fatores hereditários parciais.

\section{Conclusão}


Em vista da amplitude etiológica e dos modos de compreensão e manifestação do Transtorno de Personalidade Borderline (TPB) buscou-se, através da realização do presente estudo, sistematizar as principais características e alterações neuropsicológicas associadas à presença do transtorno. De antemão, foi possível concluir que há uma gama de alterações a nível cognitivo e a nível biológico - alterações na região límbica e em outras regiões do cérebro.

Entre as principais alterações cognitivas encontradas, estão: menor funcionamento adaptativo; alterações na memória, na atenção, nas tomadas de decisão, nos processos autorreferenciais, nas respostas ao estresse e no processamento emocional.

Já referente às alterações da região límbica, destacaram-se alterações no âmbito da amígdala, hipocampo e hipotálamo. As principais alterações na amígdala citadas foram: aumento das ativações em contraste com a redução da ativação; diminuição do volume contrastando com o aumento do volume; redução significativa da massa cinzenta e mudanças na conectividade funcional. No hipocampo: redução do volume; baixo volume da massa cinzenta e aumento da ativação no hipocampo esquerdo na exposição a estímulos negativos. E no hipotálamo, foi encontrado apenas o aumento do volume.

No que se refere às alterações em outras regiões do cérebro, as principais alterações encontradas foram: alterações nas regiões de controle pré-frontal; maior concentração de $\mathrm{N}$ acetilasparto e glutamato; alteração na região orbitofrontal, dorso-medial e dorsolateral préfrontal; e o aumento da ativação no córtex cingulado posterior esquerdo. Além disso, embora com menor frequência, mas com demasiada importância, também foram encontradas alterações na frequência cardíaca, assim como fatores hereditários associados ao TPB que abrem portas para o desenvolvimento de outros estudos.

Assim, a partir do mapeamento dessas características e alterações neuropsicológicas do TPB, tornou-se possível organizar em um único local os principais achados que, de algum modo, espera-se que elucidar e ampliar o debate a respeito da patologia do borderline, bem como facilitar a busca dessas informações na literatura brasileira e, principalmente, contribuindo para essa discussão.

Por fim, a limitação desse estudo encontra-se no fato das produções, em sua maioria, apresentarem as alterações/características biológicas separadamente de seus correspondentes cognitivos, não obstante alguns estudos tenham feito menção a essas 
correspondências, tal como foram expostas ao longo do desenvolvimento do presente trabalho. Além disso, em alguns momentos, esse estudo sugeriu algumas correspondências entre os achados mencionados. Portanto, tornam-se necessárias maiores investigações em busca de melhores evidências científicas. Enfim, enquanto recomendação, destaca-se a importância de pesquisar e ampliar a discussão no âmbito neuropsicológico do Transtorno de Personalidade Borderline (TPB) na literatura brasileira, sobretudo devido à evidente escassez dessa discussão na literatura e a falta de dados consideráveis acerca da correspondência sobre essas características e alterações neuropsicológicas e o TPB em uma amostra populacional brasileira, abrindo-se, então, caminhos para novas pesquisas.

\section{Referências}

American Psychiatric Association. (2014). Manual diagnóstico e estatístico de transtornos mentais: DSM-5. 5 ed. Porto Alegre: Artmed.

Bertsch, K., Hillmann, K. \& Herpertz, S, C. (2018). Behavioral and Neurobiological Correlates of Disturbed Emotion Processing in Borderline Personality Disorder. Psychopathology, 5I (2), 76-82. http://dx.doi.org/Io.II59/ooo487363.

Chapman, J., Jamil, R. T. \& Fleisher, C. (202I) Borderline Personality Disorder. In StatPearls. StatPearls Publishing.

Dalgalarrondo, P. (2019). Psicopatologia e semiologia dos transtornos mentais. $3^{\underline{a}}$ ed. Porto Alegre: Artmed.

Kaess, M., Parzer, P., Koenig, J., Resch, F. \& Brunner, R. (2016). Dual-task performance under acute stress in female adolescents with borderline personality disorder. European Child ¿ Adolescent Psychiatry, 25 (9), 1027-1035. http://dx.doi.org/10.1007/s00787-016-0824-7.

Kimmel, C. L., Alhassoon, O. M, Wollman, S. C., Stern, M. J., Perez-Figueroa, A., Hall, M. G., Rompogren, J. \& Radua, J. (2016). Age-related parieto-occipital and other gray matter changes in borderline personality disorder: a meta-analysis of cortical and subcortical structures. Psychiatry Research: Neuroimaging, 251, 15-25. http://dx.doi.org/ı.1016/j.pscychresns.2016.04.005.

Krause-Utz, A., Winter, D., Niedtfeld, I. \& Schmahl, C. (2014). The latest neuroimaging findings in borderline personality disorder. Current psychiatry reports, 16 (3), 438, 2014 https://doi.org/10.1007/sir920-014-0438-z 
Krause-Utz, A. D. \& Elzinga, B. M. (2019). Neurale mechanismen van dissociatie: implicaties voor borderlinepersoonlijkheidsstoornis [Neural mechanisms of dissociation: implication for borderline personality disorder]. Tijdschrift voor psychiatrie, 6I (4), 267-275.

Lamers, A., Toepper, M., Fernando, S. C., Schlosser, N., Bauer, E., Woermann, F., Driessen, M. \& Beblo, T. (2019). Nonacceptance of negative emotions in women with borderline personality disorder: association with neuroactivity of the dorsal striatum. Journal of psychiatry d neuroscience: JPN, 44 (5), 303-312. https://doi.org/ı0.1503/jpn.180077.

Luria, A, R. (198I). Fundamentos de neuropsicologia. Tradução de Juarez Aranha. Rio de Janeiro: Livros técnicos e científicos.

O'neill, A. \& Frodl, T. (2012). brain structure and function in borderline personality disorder. Brain Structure dr Function, 217, 767-782. https://doi.org/I0.1007/soo429-012-0379-4

Perez-rodriguez, M. M., Bulbena-cabré, A., Bassir Nia, A., Zipursky, G., Goodman, M. \& New, A. S. (2018). The Neurobiology of Borderline Personality Disorder. The Psychiatric clinics of North America, 4I (4), 633-650. https://doi.org/10.1016/j.psc.2018.07.012

Rossi, R., Lanfredi, M., Pievani, M., Boccardi, M., Rasser, P.E, Thompson, P.M., Cavedo, E., Cotelli, M., Rosini, S. \& Beneduce, R. (2015). Abnormalities in Cortical Gray Matter Density in Borderline Personality Disorder. European Psychiatry, 30 (2), 221-227. http://dx.doi.org/ıo.1016/j.eurpsy.2014.II.0o9.

Ruocco, A, C. \& Bahl, N. (2014). Material-specific discrepancies in verbal and visual episodic memory in borderline personality disorder. Psychiatry Research, 220 (I-2), 694-697. http://dx.doi.org/ı.1016/j.psychres.2014.07.oro.

Ruocco, A, C. \& Carcone, D. (2016). A Neurobiological Model of Borderline Personality Disorder: systematic and integrative review. Harvard Review Of Psychiatry, 24 (5), 311-329. http://dx.doi.org/Io.1097/hrp.ooooooooooooor23.

Scherpiet, S., Herwig, U., Opialla, S., Scheerer, H., Habermeyer, V., Jäncke, L. \& Brühl, A, B. (2015). Reduced neural differentiation between self-referential cognitive and emotional processes in women with borderline personality disorder. Psychiatry Research: Neuroimaging, 233(3), 314-323. http://dx.doi.org/I0.1016/j.pscychresns.2015.05.008.

Schmahl, C., Herpertz, S. C., Bertsch, K., Ende, G., Flor, H., Kirsch, P., Lis, S., MeyerLindenberg, A., Rietschel, M. \& Schneider, M. (2014). Mechanisms of disturbed emotion processing and social interaction in borderline personality disorder: state of knowledge and research agenda of the german clinical research unit. Borderline Personality Disorder And Emotion Dysregulation, I (I), I2. http://dx.doi.org/I0.I186/205I-6673-I-I2.

Schmitz, M. Müller, L. E., Schulz, A., Kleindienst, N., Herpertz, S. C. \& Bertsch, K. (2020). Heart and brain: cortical representation of cardiac signals is disturbed in borderline 
personality disorder, but unaffected by oxytocin administration. Journal of Affective Disorders, 264, 24-28. http://dx.doi.org/ı.1016/j.jad.2019.11.139.

Schreiner, M. W., Klimes-Dougan, B. Mueller, B. A., Nelson, K. J., Lim, K. O. \& Cullen, K. R. (2019). Neurocircuitry associated with symptom dimensions at baseline and with change in borderline personality disorder. Psychiatry Research: Neuroimaging, 290, 5865. http://dx.doi.org/I0.1016/j.pscychresns.2019.07.001.

Schulze, L., Grove, M., Tamm, S., Renneberg, B. \& Roepke, S. (2019). Effects of transcranial direct current stimulation on the cognitive control of negative stimuli in borderline personality disorder. Scientific reports, 9 (I), 332. https://doi.org/ıo.1038/s41598or $8-37315^{-x}$

Sebastian, A., Jung, P., Krause-Utz, A., Lieb, K., Schmahl, C. \& Tüscher, O. (2014). Frontal Dysfunctions of Impulse Control - A Systematic Review in Borderline Personality Disorder and Attention-Deficit/Hyperactivity Disorder. Frontiers In Human Neuroscience, 8, 698. http://dx.doi.org/ro.3389/fnhum.2014.00698.

Souza, M. T., Silva, M. D. \& Carvalho, R. (2010). Revisão integrativa: o que é e como fazer. Einstein (São Paulo), I (I), I02-106. https://doi.org/ı0.1590/si679-45082o10rwir34. Press.

Stirling, J. \& Elliott, R. (2008). Introducing Neuropsychology. New York: Psychology

Vai, B., Cazzetta, S., Scalisi, R., Donati, A., Bechi, M., Poletti, S., Sforzini, L., Visintini, R. \& Maffei, C. Benedetti, F. (202I). Neuropsychological deficits correlate with symptoms severity and cortical thickness in Borderline Personality Disorder. Journal Of Affective Disorders, 278, I8I-I88. http://dx.doi.org/ıo.1016/j.jad.2020.09.060.

Zaehringer, J., Ende, G., Santangelo, P., Kleindienst, N., Ruf, M., Bertsch, K., Bohus, M., Schmahl, C. \& Paret, C. (2019). Improved emotion regulation after neurofeedback: a single-arm trial in patients with borderline personality disorder. Neuroimage: Clinical, 24, 102032. http://dx.doi.org/ro.1016/j.nicl.2019.102032.

Zegarra-Valdivia, J. A. \& Vilca, B. N. C. (2019). Social cognition and executive function in borderline personality disorder: evidence of altered cognitive processes. Salud Mental, 42 (I), 33-42. http://dx.doi.org/I0.1771I/sm.0185-3325.2019.005. 